\section{Hannes Haas}

\section{Empirischer Journalismus}

Verfahren zur Erkundung gesellschaftlicher Wirklichkeit

Köln: Böhlau Verlag 1999. - 610 S.

ISBN 3-205-99031-5

Ein verblüffender Titel: Wir kennen die empirische Journalismusforschung, aber inwiefern kann der Journalismus selbst empirisch, also mit wissenschaftlichen Methoden vorgehen? Oder handelt es sich hier um einen Empiriebegriff, der vom Wissenschaftssystem abgekoppelt ist, also um Alltagserfahrung? Beide Vermutungen sind falsch. Eine dritte Position ist möglich, wenn der Alleinvertretungsanspruch der Wissenschaft für Empirie bestritten wird. Dazu ist es erforderlich, Empirie in verschiedene Typen oder Formen einzuteilen. Empirischer Journalismus hat nicht nur mit Erfahrung zu tun, sondern ist Deutung von Wirklichkeit, die aus zwei Schritten besteht: der Themenselektion und der anschließenden Recherche als deren handlungsbezogene Überprüfung. Auf der einen Seite unterscheidet sich wissenschaftliche von journalistischer Empirie, weil Erstere nach Verallgemeinerung ihrer Aussagen und letzterer nach Konkretisierung und Einzelfallbezogenheit strebt. Auf der anderen Seite verweist Haas auf die Parallelen zwischen Sozialwissenschaft und Journalismus, etwa am Beispiel der Sozialreportage oder des Interviews. Danach ist empirischer Journalismus „ein der Wissenschaft vorgelagertes Erkundungs- und Erkenntnissystem der Gesellschaft" (S. 16).

Empirischer Journalismus ist jedoch kein neues Berichterstattungsmuster, sondern kommt in jedem Berichterstattungsmuster vor. Mithilfe einer komparatistischen Vorgehensweise können Sozialwissenschaft, Journalismus und Literatur miteinander verglichen werden.

Dies erfordert nach Haas eine andere Definition von Journalismus als etwa eine systemtheoretische, denn Journalismus wird über seine autonome, kreative, unkonventionelle und engagierte Leistungen bestimmt. Damit ist zwar keine Rückkehr zum Journalismus der großen publizistischen Persönlichkeiten à la Dovifat beabsichtigt, aber der Schwerpunkt wird auf einen individuell autonomen Journalismus verlagert. Mit etwas Getöse wird eine Abkehr von der vermeintlich herrschenden systemtheoretischen Lehre in der Journalismusforschung postuliert, die durch verstehens- und verständigungsorientierte Ansätze ersetzt werden soll. Ziel dieser Neuorientierung ist die Bereicherung der Qualitätsdebatte im Journalismus.

Haas übt im darauf folgenden theoretischen Teil zuerst Kritik am Kritischen Rationalismus, weil die zunehmende Genauigkeit und Spezialisierung der Theorien und Methoden zur Entfernung von der sozialen Wirklichkeit führten. Besonders hart geht er mit der Systemtheorie ins Gericht, deren Entpersonalisierung und Entindividualisierung dem modernen Journalismus nicht gerecht würde. Besser weg kommt der Konstruktivismus, weil er stärker das Individuum in den Fokus seiner Forschung stellt. Allerdings lehnt Haas die radikale Variante im Anschluss an Saxers Kritik ab. Als für den Journalismus angemessene Theorieentwürfe werden die Gesellschaftstheorie von Jürgen Habermas sowie die Cultural Studies aufgeführt.

Dieser Theorieteil der breit angelegten Studie ist allerdings streckenweise ärgerlich, und dies aus mehreren Gründen. Zum einen werden die kritisierten Ansätze alles andere als gut dargestellt und enthalten eine Fülle von Irrtümern: Gegen die Systemtheorie (Luhmanns Variante ist gemeint) wird Münchs an Parsons angelegter Entwurf angeführt. Weischenbergs Adaption der Systemtheorie wird als gemäßigte Version des Funktionalismus im Sinne von Parsons gekennzeichnet. Weiß Haas denn nicht, dass Parsons die wesentlich konservativere, um nicht zu sagen borniertere Systemtheorie vertritt? Weiß Haas ferner nicht, dass Münch ebenfalls ein Systemtheoretiker ist, und zwar einer, der sich auf Parsons beruft? Eine Annäherung an Habermas' Theorie des kommunikativen Handelns sehe ich darin nicht - ganz abgesehen davon, dass Haas den Eignungsnachweis dieser Theorie für seinen Gegenstandsbereich schuldig bleibt.

Zum anderen ist der gesamte theoretische Teil alles andere als originell, sondern nur referierend. Sogar die Kritik an den vorgestellten Ansätzen ist weitestgehend entlehnt, häufig von Baum - wodurch sie in der Sache natürlich nicht entwertet wird. Man freut sich allerdings noch nicht einmal über Zustimmung: So versucht Haas, den Radikalen Konstruktivismus gegen Luhmanns Systemtheorie auszuspielen, und lobt die Betonung der individuellen Autonomie im Konstruktivismus. Dieser Beifall kommt jedoch von der falschen Seite, denn der konstruktivistische Autonomiebegriff hat mit 
Langenbuchers Konzept des autonomen Journalismus, auf den sich Haas stützt, nicht viel gemeinsam. Langenbuchers Autonomieverständnis scheint dem der Autonomen ( $j a$, die mit den Hasskappen sind gemeint!) zu entsprechen, impliziert dieser Autonomiebegriff doch, dass bestimmte gesellschaftliche Bereiche - hier der Journalismus - von politischen, ökonomischen und sonstigen Zwängen in quasi „befreiten $\mathrm{Zo}_{\mathrm{o}}$ nen" existieren könnte. Dies entspricht jedoch eher dem Zustand der Autarkie, als dass es etwas mit Autonomie (im systemtheoretischen und im konstruktivistischen Sinn) zu tun hätte.

Dass schließlich auch noch die Kräfte des Marktes ausgerechnet als Kronzeugen für eine individualistische Journalismustheorie herangezogen werden (vgl. S. 95), ist dann vollends verblüffend und steht in einem offenen Kontrast zur mehrfach herausgestellten kulturellen Leistung des Journalismus.

Nach dem Theorieteil gefällt mir das Buch wesentlich besser. Es folgen „Strategien der Wirklichkeitserkundung “ und „Methoden und Programme des Empirischen Journalismus“, in denen journalistische Berichterstattungsmuster und Darstellungsformen dargestellt, illustriert und analysiert werden. Die historische Ableitung öffnet den Blick dafür, a) dass diese Formen nicht einfach so bestehen, sondern sich historisch entwickelt haben, und b) dass viele Pionierleistungen bereits in Zeiten erbracht wurden, als der Journalismus gesellschaftlich noch nicht so etabliert war wie heute und deutlich mehr Zwängen unterworfen war. Diese beiden Teile gehen auch weit über das übliche Lehrbuchwissen hinaus. Zweifelsohne werden hier die Leistungen, zu denen Journalismus imstande ist, ins beste Licht gestellt. Und es ist auch begrüßenswert, einmal die Sahnestücke des Journalismus zu dokumentieren und $\mathrm{zu}$ würdigen, statt immer nur die Probleme und Skandale zu thematisieren. Die häufigeren Rückbezüge auf die theoretischen Argumente im vorigen Teil sind allerdings nicht zwingend, vielleicht sogar überhaupt nicht notwendig.

Im abschließenden Epilog fasst Haas die wichtigsten Aspekte zusammen. In der Informationsgesellschaft kann sich Journalismus nur dadurch profilieren, dass er die Geschehnisse kritisch reflektiert. Objektiver Informationsjournalismus allein ist nicht ausreichend - und war er auch nie, denn neben ihm existierten immer auch andere Berichterstattungsmuster. Wenn Journalismus in diesem Sinn empirisch ist, kann er als legitimes Konstruktionssystem (sic!) gesellschaftlicher Wirklichkeit anerkannt werden und hat seinen Platz neben anderen "Journalismen" (S. 382 f.), wobei ich den Plural wieder nicht verstehe, oder ist hier doch wieder Berichterstattungsmuster gemeint, was im Widerspruch zum vorher Gesagten stünde? Haas befürwortet eine journalistische Rolle als Bricoleur und Flaneur, beides Denkfiguren der Moderne. Allerdings fehlt mir die Abgrenzung zur Postmoderne, die ebenfalls eine Art Bricoleur kennt, diesen aber als Patchworker interpretiert. Und mir missfällt der flanierende - um nicht zu sagen leichtfertige und überhebliche Umgang mit Theorien. Das ist postmodernes Geschichte-Erzählen, aber sollten wir Wissenschaft wirklich so verstehen und darauf reduzieren?

Armin Scholl

\section{Uwe Hasebrink/Patrick Rössler (Hrsg.) Publikumsbindungen}

Medienrezeption zwischen Individualisierung und Integration

München: R. Fischer 1999 - 193 S.

(Reihe Angewandte Medienforschung; 12)

ISBN 3-88927-253-3

Die in den letzten Jahren verstärkt geführte wissenschaftliche Auseinandersetzung zum Phänomen der Individualisierung war durch einen typischen Verlauf charakterisiert. Angeregt aus der Soziologie (vgl. Beck, Schulze) führte die Debatte in der Medien- und Kommunikationswissenschaft sehr schnell zu einer intensiven Beschäftigung mit dem Begriff, wobei dieser nicht selten als universelles Erklärungsmodell für generelle Entwicklungen in der $\mathrm{Me}$ diengesellschaft herangezogen wurde. Erst danach folgte eine Phase der Differenzierung und Bewertung seines Erklärungspotenzials auf unterschiedlichen theoretischen Niveaus.

Der vorliegende Band, der die im Januar letzten Jahres abgehaltene Arbeitstagung der Fachgruppe "Rezeptionsforschung“ der DGPuK dokumentiert, legt nunmehr eine Reihe großteils empirischer Untersuchungen vor, die sich mit der Medienrezeption zwischen den beiden Polen der Individualisierung und Integration beschäftigen. Bereits im Vorwort liefern die Herausgeber dabei den entscheidenden 